\title{
Development of Atom Probe Tomography with In-situ STEM Imaging and Diffraction
}

\author{
B. P. Gorman, * J. D. Shepard, ${ }^{* *}$ R. Kirchhofer, ${ }^{*}$ J. D. Olson, ${ }^{* *}$ T. F. Kelly** \\ * Department of Metallurgical and Materials Engineering, Colorado School of Mines, Golden, CO \\ 80401 \\ ** Cameca Instruments, Inc., 5500 Nobel Drive, Madison, WI, 53711
}

Atom probe tomography (APT) allows for compositional analysis of materials at the atomic scale through imaging time-of-flight mass spectroscopy. 3-D volume renderings of atomic-scale chemistry are possible following reconstruction algorithms. Unfortunately, a-priori information about the electric field necessary for evaporation of individual ions is not well documented for many materials, but is required for reconstruction. In order to generate such data, quantification of the specimen geometry and internal interfaces with nominally $1 \mathrm{~nm}$ precision is necessary. Ex-situ monitoring of atom probe specimens has been completed previously in some detail using HRTEM, STEM, tomography, EDS, and EELS [1-3], however, difficulties exist in the transfer of specimens between instruments. In order to solve this, a combined STEM and APT system is currently being engineered and constructed.

The system is based on a Cameca LEAP 4000X Si atom probe tomography platform, with the integration of a UHV compatible electron column and detectors. Due to the required UHV conditions for atom probe tomography, the selection of a suitable electron column with nominally 1 $\mathrm{nm}$ spatial resolution is somewhat limited. However, a $30 \mathrm{keV}$ column with the necessary requirements has been engineered to one of the optical alignment ports of the APT (Fig. 1). In the current engineering design, the specimen working distance is minimized in order to achieve the highest optical spatial resolution without interfering with the ion flight paths.

Electron detection will be undertaken using 3 separate detectors. First, a standard Everhart-Thornley scintillator detector will be used for secondary electron imaging and positioning of the specimen with high accuracy and repeatability. Second, a solid state detector will be used as a bright field / dark field transmission electron detector for high speed and high resolution STEM imaging, an example of which is given in Fig. 2. Finally, a third phosphor screen will be used as a diffraction detector, enabling STEM-diffraction monitoring of the specimen during the APT experiment, similar to EBSD mapping. Diffraction patterns will be utilized as internal reference standards in order to determine the interplanar spacing of the atomic planes, as well as their directions with respect to the ion-detector trajectories. The working distance of the specimen to the phosphor screen was engineered such that a wide range of materials could be analyzed at $30 \mathrm{keV}$ accelerating voltages. The possibilities for dynamic experiments based on laser pulse pump-electron diffraction probe and subsequent atom probe tomography are possible using this arrangement [4].

References

[1] B. P. Gorman, D. Diercks, N. Salmon, E. Stach, G. Amador, C. Harfield, Microscopy Today, 16 (4), 42-48 (2008).

[2] B. P. Gorman, A. Puthucode, D. R. Diercks, M. J. Kaufman, Mat. Sci. \& Tech., 24 (6) 682-688 (2008). 
[3] B. P. Gorman, D. Diercks, M. Kaufman, R. Ulfig, D. Lawrence, K. Thompson, and D. J. Larson, Microscopy and Microanalysis, 12 (Suppl. 2) (2006) 1720CD.

[4] This research is supported by the National Science Foundation under contract \# 1040456

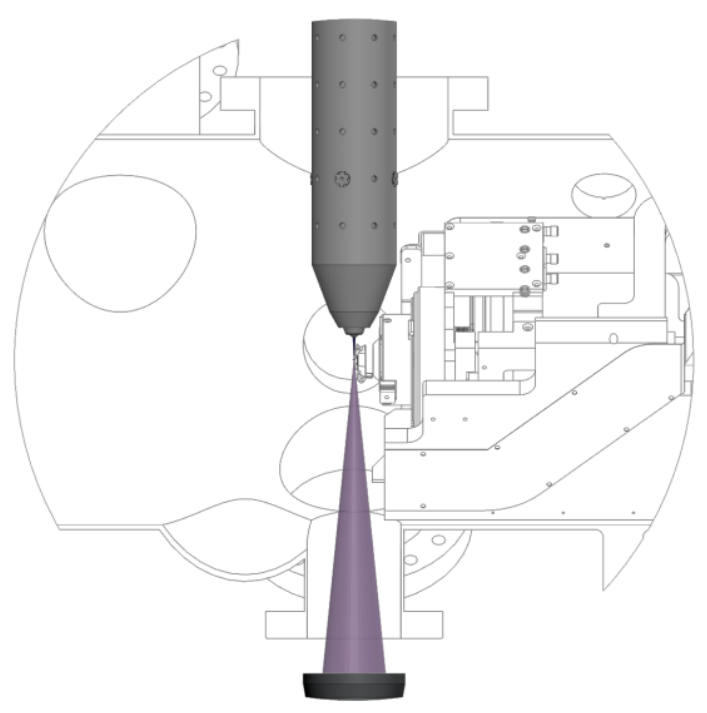

FIG. 1. Schematic illustrating the incorporation of a STEM-diffraction experiment into a LEAP 4000X Si. The STEM column is shaded gray with electron diffraction trajectories illustrated in purple.

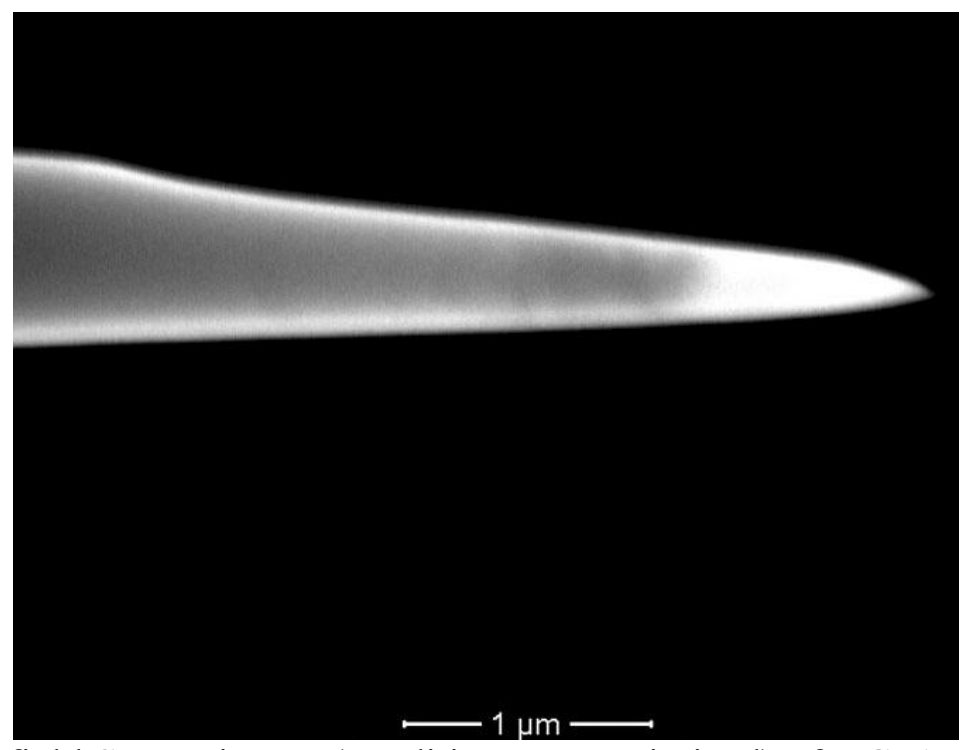

FIG. 2. $30 \mathrm{keV}$ dark field STEM image (conditions not optimized) of a GaAs atom probe specimen illustrating thickness and diffraction contrast. 